\title{
An Interactive Multimedia Business Game
}

\author{
Lynda Hardman, Guido van Rossum
}

CWI

\begin{abstract}
Aart van Bolhuis
Mediatrix

The playing of computer-based business games has changed little with recent technological improvements. Teams make decisions which are written down on paper and handed to a tutor who then has to enter these into the computer. To make it a richer and more real-life experience we have brought a business simulation into a multimedia environment. In order to create a sense of the passing of time the simulation is continuous, and players can enter input values throughout the game. Important parameters, such as product sales, are displayed as continuously updated graphs. Background information such as news items is provided in multimedia form. Players also have direct access to online tools, such as spreadsheets, from the game environment. We describe the prototype built to illustrate our approach.
\end{abstract}

KEYWORDS: continuous simulation; interactive simulation; multimedia business game; multimedia management game

In simulation-based business games teams compete with each other in a simulated business world, see for example (Burgess, 1989; Bryant \& Corless, 1986; Gentry, 1990). The educational goals of these games vary depending on the specific subject area that they address. General areas in which players can gain experience include applying their financial and marketing skills. A characteristic of these business games is that the actions of the players do not impact on the real world - the worst thing that can happen is that players score badly. They are also able to experience working as part of a team and benefit from valuable feedback on their performance from a tutor. The motivation for our work is to enhance the gaming environment, through rich interactivity, both for the players and the tutor, while preserving the original educational goals of the business game.

The traditional set-up for this type of game is that a simulation model, along with a set of decisions from each of the teams, determines which team sells most 
product in a period and how much profit each makes. The teams spend a fixed amount of time making their decisions and submit these on decision sheets. A course coordinator submits this information to the simulation program, which then runs through the data and produces the results. This process has the bare minimum of interaction with the computer. Suggestions in the literature have already been made for making improvements in the degree of interactivity with this type of game, for example the following can be found among the recommendations for future directions given in (Loveluck, 1989) (paraphrased).

(1) Allow the instructor to alter the coefficients of equations used within the simulation, and adjust which information can be accessed by the participants.

(2) Allow the instructor to change aspects of the game while it is running, for example by altering the inflation rate.

(3) Integrate commonly-used personal-computer tools with the game, for example spreadsheets, graph drawing facilities. Use these for input of decisions and output of results and also allow "what if" analyses.

(4) Allow different levels of decisions to be made, for example each team member has an area of responsibility but for important decisions all team members have to agree.

(5) Allow participants to enter decisions at any time rather than at the end of a set time period.

Wolfe, in (Wolfe 1993), seems to support these points. Based on a survey amongst US experts, he forecasts how business games may look in the future. Wolfe suggests that players will still work in teams, real-time play will be possible, players will have direct access to other relevant computer applications (for example spreadsheets for "what if" analyses), and decisions will be submitted interactively.

Both these sources suggest a move towards a far more interactive and responsive computer environment. In order to gain an understanding of the implications of this type of interactivity, we have taken an existing business simulation and have embedded it in an interactive multimedia information environment. The subject matter of the game remains the same but the playing experience becomes very different. We have implemented most of the suggestions from Loveluck (denoted by the numbers), as well incorporating multimedia information into a prototype game:

- The tutor can alter values of the simulation parameters (2), although not the equations used within the simulation (1).

- Applications, such as spreadsheets, can be accessed directly from the game (3). 
- Different roles in the team have their own screen (4), but the access to these is open.

- The simulation has been reimplemented to run continuously, and allows players to enter decisions at any time during the game (5).

- Characters from the simulated world have been recorded on digital video and appear from time to time to bring messages to the players (a much more extensive use of this technique can be found in (Stevens, 1989)).

Our work does not consider the educational advantages and disadvantages of management simulations. Discussions on, for example, the educational value of management simulations are given in (Keeffe et al., 1993), and on which types of simulation are more suitable can be found in (Li \& Baillie, 1993).

The prototype described in this paper was designed with help from lecturers at the Hogeschool Holland, a business school based in Amsterdam, to ensure that in adding the extra interactivity we were not detracting from the learning goals of the business game (although the authors remain responsible for the final design). At the Hogeschool Holland a business game is introduced into the curriculum at two points: once at the beginning of their course to let the students have some fun running a company, and once later in their course when they can see just how much they have learned during their courses. (Similar use of a management game is made in (Gooding \& Keys, 1990).)

The main part of this paper is dedicated to describing the prototype game, after which the current status of the prototype is reported.

\section{A Prototype Interactive Multimedia Business Game}

The prototype game supports the participation of four teams under the direction of a tutor. The tutor and teams use computers connected to the same network. Both these roles take part in the game via a set of screens (described in detail later in this section).

The game is built in a modular fashion from three basic components: the simulation, a server, and a user environment, as shown in Fig. 1. The simulation program implements the rules of the game, stores the current state of the "world" and, at regular intervals (every 5 or 10 minutes, or a game "week"), computes the new state. The calculation of the new state is based upon user input, rules within the simulation and the previous state. The simulation logs some of the output values for every iteration of the simulation. The only way of interacting with the simulation is by changing parameter values, and these changes are always sent via the server. The server passes user input to the simulation and allows users to query the state (insofar as they are allowed access). The server communicates with the user environment over the network. The user environment consists of a 


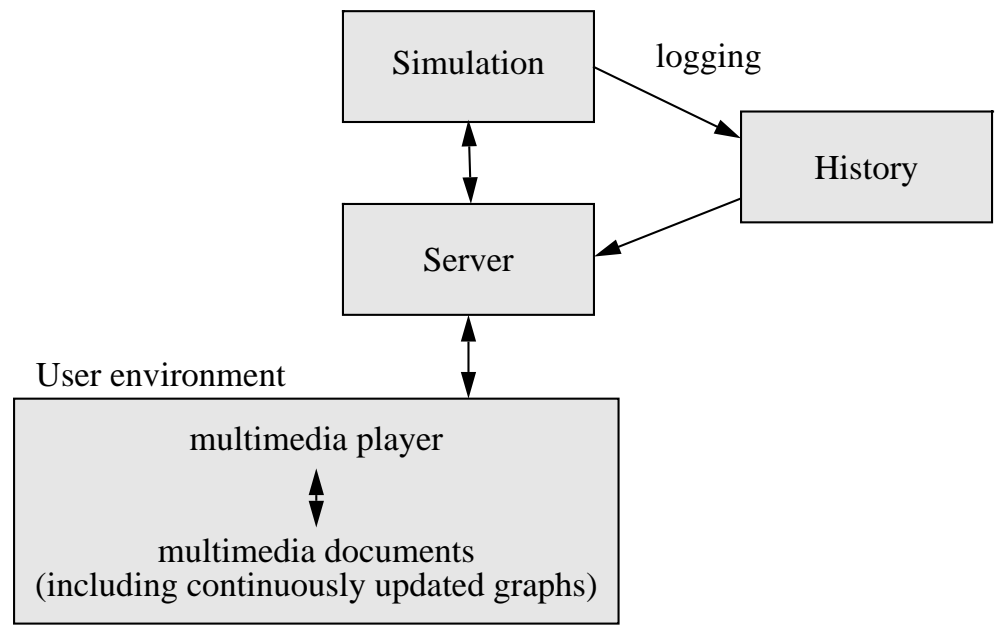

Figure 1 Structure of the game

set of multimedia documents and a general purpose multimedia player-adapted for interaction with the server from the player described in (Van Rossum et al., 1993). Input from the tutor and teams is transmitted from the user environment to the server, and can be submitted at any time. The output values from the server are used for generating, for example, graphs of values for display to the players.

\section{Game screens}

There are two kinds of game screens - the main tutor screen and the department screens for each team. The purpose of the tutor screen is to give an overview of all the simulation parameters in the game and to give the tutor access to the game screens of each team. Each team has access to five departments where different sets of decisions are taken: management, finance, sales, marketing, and production. The screens also give access to background information and other applications, and display short videos, such as a visit to the bank manager.

The department screens for the teams are each divided into 4 portions. Top left shows the values of relevant simulation parameters as continuous graphs, where appropriate, or in tabular form. Top right is used as a display area for the multimedia news items and where characters in the game (e.g. the bank manager) can address the team. Bottom left has dialogues for the teams to enter their decisions. Bottom right shows other information relevant to making the decisions, and where appropriate, gives access to external programs, such as a spreadsheet. The key to the meaning of the graphics shown in each of the example screens is given in Fig. 2. 
Volume Present position in system

Volume Link to other part of system

Send Decision Send decision to simulation

40 Numeric input by users

\begin{tabular}{|l|}
\hline Expected higher demand after hiring sales staff. \\
\hline
\end{tabular}

Figure 2 Key to graphics used in game screens

\section{Tutor screen}

The tutor cannot change the state of the simulation directly, but can change the values of the simulation parameters. Changing specific simulation parameters, for example the economic factor, can result in sending a "news item" to the teams. These items are constructed by the game designer. The tutor screen, shown below, is split into four sections. Top left gives access to all of the screens of each of the companies and the message system. In cases of emergency, the tutor is able to pause the whole simulation. Top right displays the current multimedia presentation being displayed on all the department screens, in this case a visit to the bank manager. Bottom left is a list of time-stamped decisions taken by

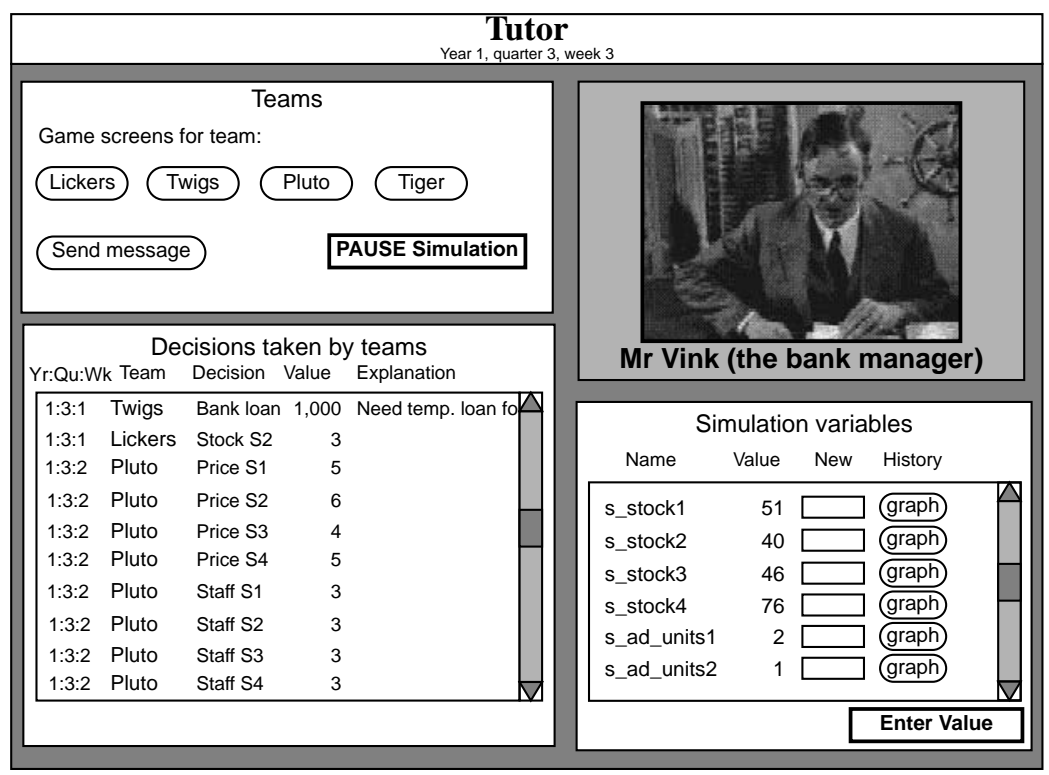


the teams. Bottom right is a complete list of all the variables which the tutor can change or view the history.

\section{Management Team}

The task of the management team is to determine the company strategy and run the company in order to achieve this strategy. High-level decisions, such as the yearly dividend for shareholders or company salaries, are taken here. First level information required for making these decisions is displayed on this screen and more detailed information can be accessed from the other department screens.

If any negotiations with "external" players, such as the unions or the board of directors (played by the tutor or as prerecorded videos), have to be carried out these are also controlled from this screen. At the end of a game year each team has to produce an annual report.

\section{Finance Department}

The finance department has to ensure the company maintains a healthy cash flow. Decisions which have to be made are the amount of loan or cash deposit, along with a reason for requesting a loan. A large amount of financial information is displayed on this screen either directly or indirectly via the use of hyper-

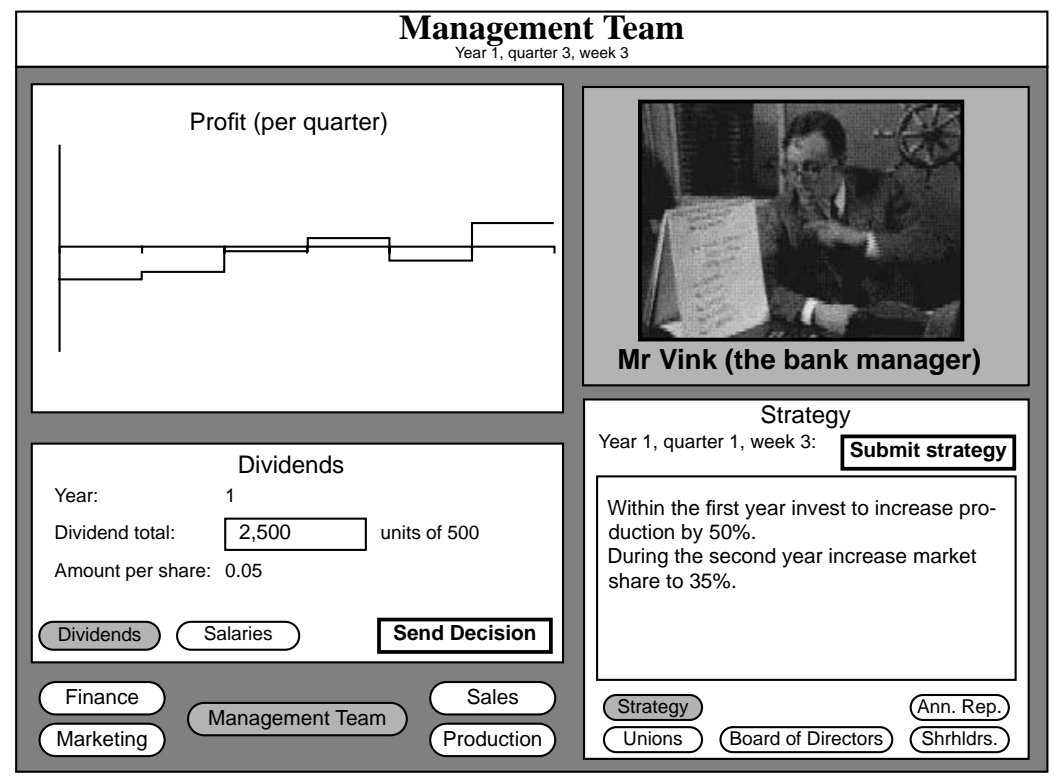




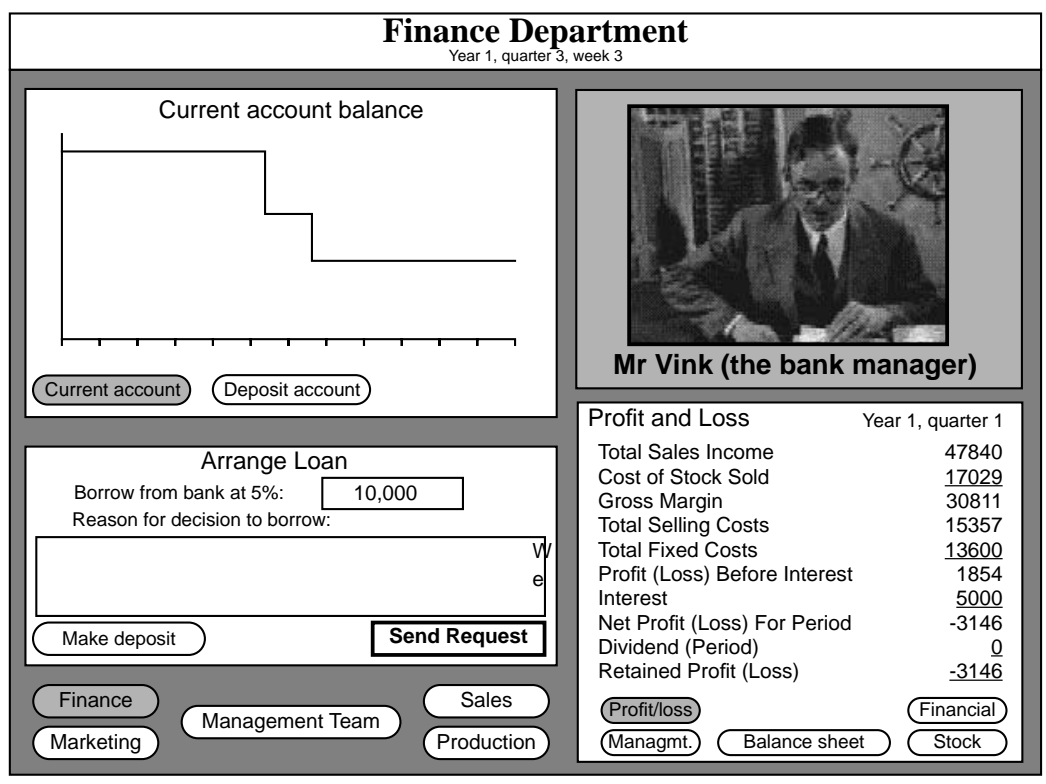

links (jumps to other parts of the information). To make the game more realistic, financial results are accumulated for a complete quarter before being accessible. Players have access to a spreadsheet from the various financial information areas (bottom right).

\section{Market Research}

Requesting and displaying market research are the tasks of this department. Companies receive free updates on the sales prices for each company per segment. They have to pay for other information, such as advertising, sales staff and detailed sales results for competitor companies and future market potential.

\section{Sales Department}

Decisions about pricing, sales staff, advertising and stock allocation to market segments are made in the sales department. 

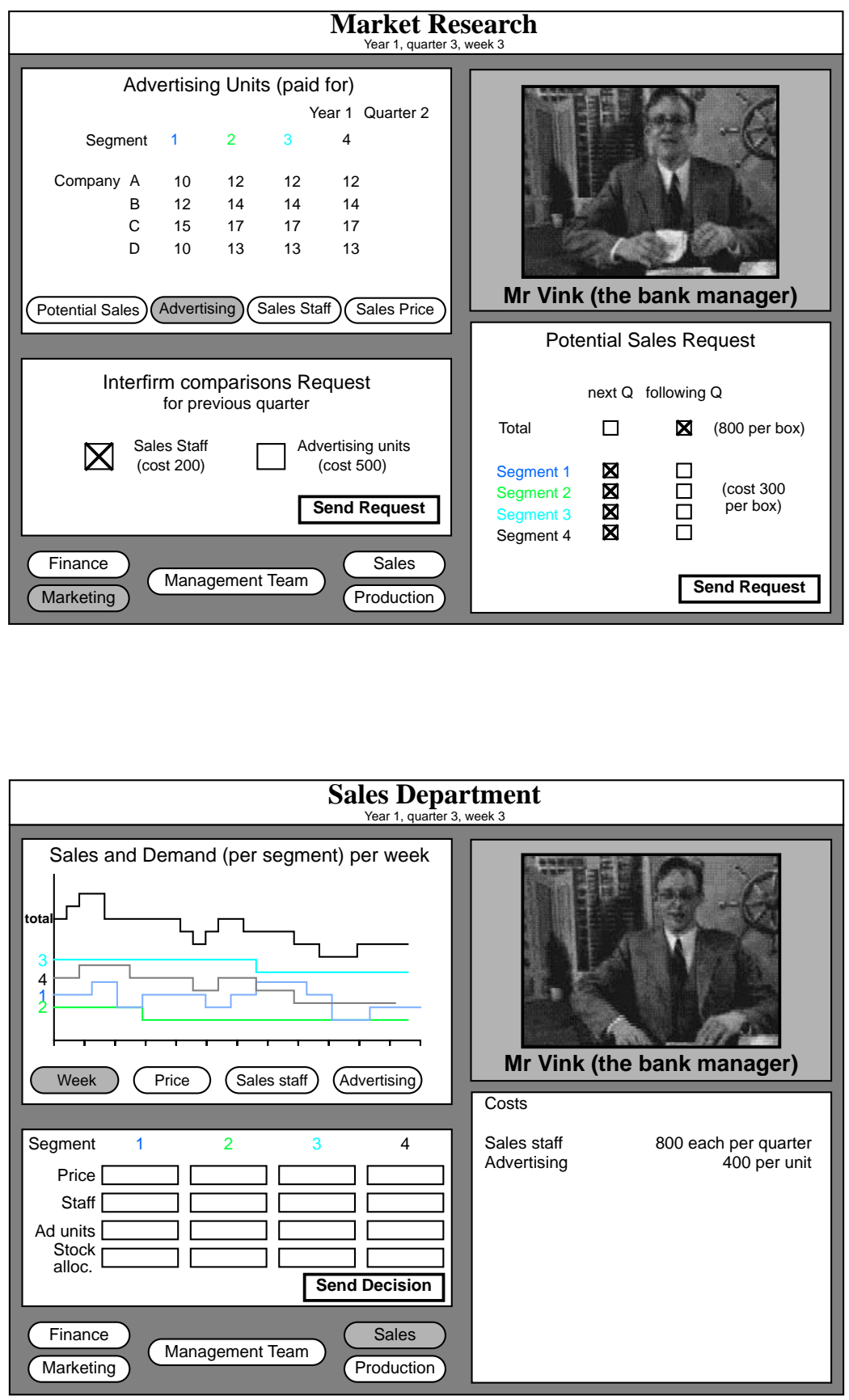


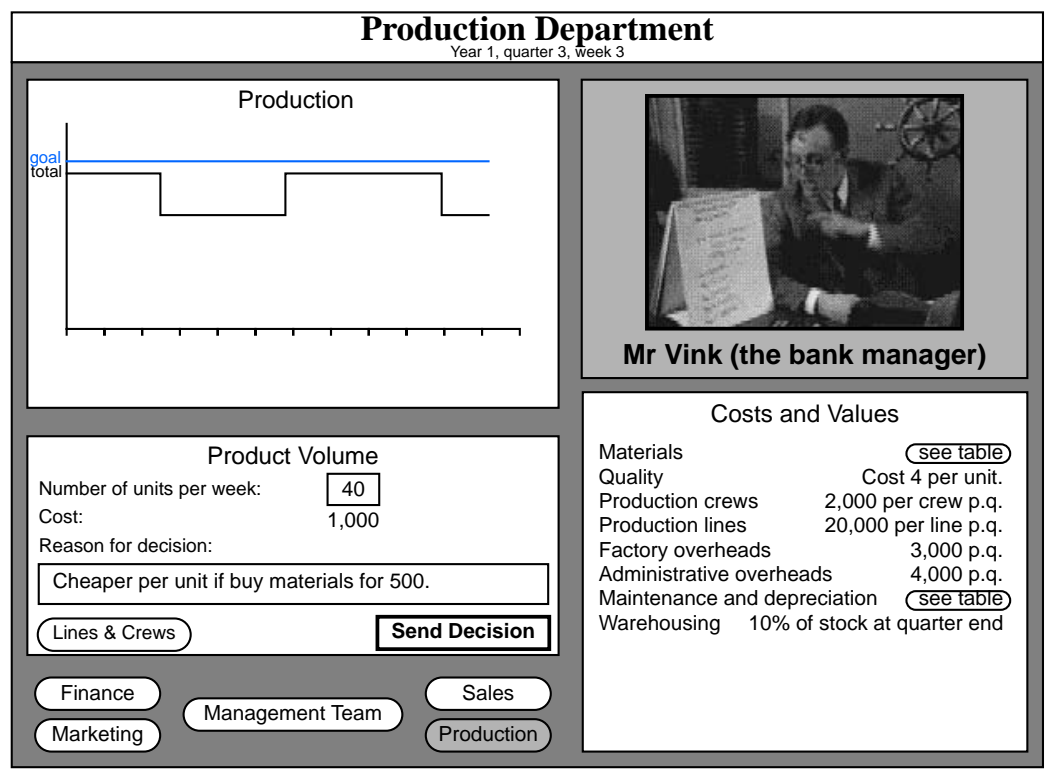

\section{Production Department}

The production department has to ensure that the sales department is supplied with sufficient product to meet predicted demand. The number of crews and production lines has to be controlled, sufficient raw materials purchased and a balance of extra stock, held in warehouses, maintained.

\section{Current Status}

We have built a prototype to demonstrate how a business simulation can be made more interactive. The prototype needs to be put on trial before any conclusions can be drawn about the pros or cons of adding interaction and multimedia to management games. The prototype runs on Silicon Graphics Indigo workstations, so that in order to make the game commercially viable it needs to be ported to a more readily available platform.

Possible enhancements to the current prototype could address a number of areas: the simulation itself could be made more complex, for example by allowing more global influences affect the game market; the tutor could be given more support, for example by having the program collect information on the teams' performance during the game; the teams could be given more support, for example by giving more integrated access to "what if" analysis tools. 
Further learning aspects could be added to the game, for example the management team could be required to assign a budget to each department as part of the second year of the game. Further use of multimedia technology could be made by adding live audio and video communication facilities among remote team members.

\section{Acknowledgements}

Jack Jansen and Sjoerd Mullender have helped with the design and implementation of the prototype. They, and the first two authors, are members of the Multimedia Kernel Systems group at CWI, headed by Dick Bulterman. Rob Macredie gave a number of helpful review comments. The initial simulation model was supplied by the Hogeschool Holland. Initial research work in the project was funded by PBTS of the Dutch department of Economic Affairs.

\section{References}

Bryant, N., \& Corless, H. (1986). The Management of Management Games. Simulation \& Games, 16(3), 99 - 112.

Burgess, T. F. (1989). The Use of Computerized Games in Business Studies. Educational and Training Technology International, 26(3), 226 - 238.

Gentry, J. W. (Ed.) (1990). Guide to Business Gaming and Experiential Learning. ABSEL (Association for Business Simulation and Experiential Learning), Nicols/GP Publishing, East Brunswick / Kogan Page, London.

Gooding, C., \& Keys, B. (1990). Introducing Executive MBA Programmes with Management Games. Journal of Management Development, 9(2), 53 - 60.

Keeffe, M. J., Dyson, D. A., \& Edwards, R. R. (1993). Strategic Management Simulations: A Current Assessment. Simulation \& Gaming, 24(3), 363 368.

Li, E. Y., \& Baillie, A. S. (1993). Mixing Case Method with Business Games: Student Evaluations. Simulation \& Gaming, 24(3), 336 - 355.

Loveluck, C. (1989). Some Conceptual Changes in Micro-Computer Based Games. Simulation \& Games, 19(4), 151 - 159.

Van Rossum, G., Jansen, J., Mullender, K. S., \& Bulterman, D. C. A. (1993). CMIFed: a Presentation Environment for Portable Hypermedia Documents. Proceedings of ACM Multimedia '93, Anaheim, CA, 183-188. 
Stevens, S. M., (1989). Intelligent Interactive Video Simulation of a Code Inspection. Communications of the ACM, 32(7), 832 - 841.

Wolfe, J. (1993). A Forecast of Business Gaming in the Year 2010. Simulation \& Gaming, 24(3), 373-375.

Lynda Hardman researches in authoring and document-structure aspects of hypermedia and multimedia, in particular providing support for creating hypermedia presentations.

Guido van Rossum's research interests include designing and implementing authoring software for hypermedia presentations, and operating system and network support for multimedia and hypermedia.

Aart van Bolhuis has considerable experience in commercial interactive video and is currently a director of Mediatrix $b v$.

ADDRESSES: LH \& GvR, CWI, CST, Kruislaan 413, PO Box 94079,

1090 GB Amsterdam, The Netherlands. Tel +31.20 .5924127 ,

fax+31.20 592 4199, email \{lynda,guido\}@cwi.nl,

WWW server http://www.cwi.nl/

$\boldsymbol{A v B}$, Mediatrix bv, Mediacentrum, Sumatralaan 45, 1217 GP Hilversum, The Netherlands 